\title{
ESTRATÉGIA E CULTURA NA INTERNACIONALIZAÇÃO DA EDUCAÇÃO SUPERIOR: A EXPERIÊNCIA DA UNIVERSIDAD DE LA INTEGRACIÓN DE LAS AMÉRICAS ${ }^{1}$
}

\author{
STRATEGY AND CULTURE IN THE INTERNATIONALIZATION OF \\ HIGHER EDUCATION: THE EXPERIENCE OF THE UNIVERSIDAD DE LA \\ INTEGRACIÓN DE LAS AMERICAS
}

\begin{abstract}
Victor Meyer $\mathrm{Jr}^{2}$ Bernardo Meyer ${ }^{3}$

Elisa Kretzer Santos 4

RESUMO: O objetivo deste estudo é examinar as estratégias utilizadas e os problemas culturais decorrentes de sua implementação nos primeiros anos de atuação da Universidad de la Integración de las Américas - UNIDA no Paraguai. Trata-se de Universidade que integra a rede de instituições do grupo educacional brasileiro Sociedade de Ensino Superior Estácio de Sá. Este trabalho caracteriza-se como um estudo de caso, de natureza descritiva. Os dados foram coletados a partir de entrevistas semi estruturadas realizadas com a totalidade dos dirigentes da organização, assim como com um ex-dirigente. A análise dos dados revelou que as principais estratégias utilizadas pela instituição foram de natureza, essencialmente, competitivas. Conflitos decorrentes de problemas culturais emergiram exigindo adaptações à cultura local como forma de materializar as estratégias, o que contribuiu para a viabilização do empreendimento. As principais conclusões indicam que o caso da UNIDA constitui-se em exemplo bem sucedido de internacionalização de um grupo educacional brasileiro. Dificuldades iniciais decorrentes de conflitos culturais resultaram em aprendizado organizacional aos gestores da organização constituindo-se em importante experiência internacional.
\end{abstract}

PALAVRAS-CHAVE: estratégia, internacionalização, educação superior

ABSTRACT: The purpose of this paper is to examine the strategies and the cultural problems related to their implementation in the early years of the Universidad de la Integración de las Américas - UNIDA in Paraguay. The University is part of a network of higher education institutions under the umbrella of a Brazilian educational group, named Sociedade de Ensino Superior Estácio de Sá. This work is descriptive in nature and is characterized as a case study. The data was collected based on semi-structured interviews with all the top administrators, as well as one former administrator of the University. The data analysis revealed that the main strategies used by the institution were essentially competitive. Cultural conflicts emerged during the implementation process which required adaptations to the local culture in order to execute the strategies and, therefore, contribute to the venture's feasibility. The main conclusions indicate that UNIDA's case is a well succeeded example of internationalization of a Brazilian educational group. The initial challenges emerged from cultural conflicts resulted in organizational learning and an important international experience for the organization.

KEY WORDS: strategy, internationalization, higher education

\footnotetext{
${ }^{1}$ Artigo Recebido em 18.05.2009. Revisado por pares em 15.12.2009. Recomendado em 08.02.2010 por Leomar dos Santos Editor. Publicado em 06.03.2010.

Organização Responsável pelo periódico: Universidade regional de Blumenau - FURB - www.furb.br/rn

${ }^{2}$ Pontíficia Universidade Católica do Paraná PUCPR- vmeyer@matrix.com.br

${ }^{3}$ Universidade Federal do Paraná - UFPR - bernardo meyer@hotmail.com

${ }^{4}$ Universidade do Estado de Santa Catarina - UDESC/ESAG - meyer@matrix.com.br
} 


\section{INTRODUÇÃO}

O processo de globalização da economia mundial tem ampliado significativamente os fluxos de capitais, pessoas, bens e serviços. As fronteiras e as distâncias entre os países se encurtaram em razão dos avanços e do barateamento das comunicações e dos transportes. Ampliaram-se os mercados e, com eles, surgiram novas oportunidades e ameaças.

A expansão do mercado, a nível global, tem desencadeado negociações que envolvem diferentes aspectos, como moeda, idioma, cultura e legislação, antes negligenciados pelos empresários. Quando uma empresa decide se internacionalizar, as estratégias adotadas necessitam ser adaptadas ou mesmo recriadas para adequar a organização às especificidades de um novo mercado competitivo e global.

As práticas gerenciais bem sucedidas utilizadas no país de origem nem sempre são garantia de um desempenho eficaz no mercado global. Dentre os vários aspectos importantes que influenciam diretamente o êxito das operações internacionais destaca-se a cultura dos países e regiões.

A cultura não influencia somente o comportamento do mercado, mas também o relacionamento interpessoal no ambiente organizacional. Administrar diferentes comportamentos, atitudes, visões de mundo e hábitos é um dos grandes desafios das empresas que ingressam no mercado internacional. O conhecimento do conjunto de comportamentos, crenças, atitudes, valores e hábitos que caracterizam uma população podem ser determinantes para o sucesso ou o fracasso das negociações.

As organizações de educação superior que ingressam no mercado internacional estão sujeitas às mesmas condições. A internacionalização de instituições de educação superior já é uma realidade, observando-se várias iniciativas de grupos educacionais se movimentando em direção a novos mercados.

O objetivo deste trabalho é examinar as estratégias utilizadas pela Universidad de la Integración de las Américas, instituição de educação superior ligada ao grupo educacional brasileiro Sociedade de Ensino Superior Estácio de Sá, e os problemas culturais decorrentes de sua implementação, nos primeiros anos de atuação, no Paraguai.

\subsection{Globalização e Negócios Internacionais}

A globalização é um fenômeno que vem impulsionando a realização de negócios internacionais em todas as partes do mundo. Trata-se de um processo que apresenta várias dimensões como econômica, social, política, cultural, legal e tecnológica. A globalização envolve também interesses de governos, grupos econômicos e sociedades, afetando, de forma distinta, regiões, países, grupos e indivíduos.

Ao estudar a globalização, Bhagwati (2004) a destaca como sendo a integração de economias nacionais em uma economia internacional por meio de comércio, investimento direto estrangeiro, investimentos especulativos, fluxos internacionais de pessoas e também fluxos de tecnologia. Para Gray (1999, p. 77), a globalização apresenta um forte componente tecnológico ao defini-la como "a difusão mundial das modernas tecnologias de produção industrial e de comunicação de todos os tipos através das fronteiras”. 
Uma definição mais genérica para o termo é dada por Bassi (1997, p.29), que o conceitua como sendo "um processo de integração mundial que está ocorrendo nos setores de comunicações, economia, finanças e nos negócios". Para este autor, o elemento chave do processo de globalização é a integração no âmbito internacional. Pode-se assim inferir que um dos importantes fatores do processo de globalização é o aumento de integração entre os vários atores internacionais.

A expansão do intercâmbio entre países, organizações e pessoas tem levado a uma maior intensidade nas trocas e uma maior interdependência entre os mesmos. Ohmae (2006) explica que esta interdependência resulta do fato das fronteiras nacionais serem, atualmente, menos restritivas do que no passado em decorrência dos avanços tecnológicos e dos acordos internacionais, principalmente na área do comércio.

Ao se referir à interdependência mundial, Gray (1999) reforça o entendimento de que a globalização provoca uma integração de quase todas as economias. O mesmo autor exemplifica que países, como a Coréia do Norte, procuram isolar suas economias das demais, mas, ao fazê-lo, geram um custo econômico e social elevado.

Diante do processo de globalização ocorre um crescente desenvolvimento das relações comerciais entre os países. Para tanto, as empresas e as nações necessitam preparar suas economias para poderem tirar proveito do comércio mundial. Assim, intensificam-se as trocas internacionais, principalmente por meio de exportação, importação e investimentos internacionais. No entendimento de Parker (1999) as organizações não são simplesmente afetadas pela globalização, mas suas atividades combinadas estimulam, facilitam e sustentam este processo.

A globalização não se constitui em processo neutro uma vez que implica em transformações trazendo benefícios para determinados grupos e setores, assim como problemas e dificuldades para outros. Naím (2006) ressalta que a globalização trouxe novos hábitos, novos costumes, novas expectativas, novas possibilidades e novos problemas. Bhagwati (2004), por sua vez, ao abordar problemas decorrentes da globalização destaca que esse processo tem sido foco de intermináveis controvérsias suscitando paixões hostis e violentos protestos.

Dentre as várias dimensões que integram a globalização está a cultura que cada vez mais está presente nos negócios internacionais. $\mathrm{O}$ entendimento das variâncias culturais tem se constituído em fator estratégico para o sucesso das organizações que se lançam à conquista de mercados internacionais.

\section{CULTURA E NEGÓCIOS INTERNACIONAIS}

A cultura é um importante fator a ser analisado pelas organizações que atuam no mercado internacional. Com a intensificação das trocas internacionais provocadas pela globalização, percebe-se o crescente impacto dos fatores culturais nas negociações. As culturas das diferentes nações muitas vezes representam um fator dificultador nas relações de negócios internacionais por influenciar formas diferenciadas de se compreender uma realidade. 
Países e regiões do mundo apresentam culturas que os diferenciam entre si. Ao se examinar os diversos aspectos que distinguem as regiões e países percebe-se a existência de elementos próprios que caracterizam cada cultura. (MEYER JR., MEYER, MURPHY, 2006). Embora se possa perceber uma tendência para certa padronização de valores e comportamento, continua a existir diferenças significativas entre países e regiões.

O conceito de cultura é explorado diferentemente pelos autores da área. Hofstede (1984) define cultura como uma programação coletiva de mentes que diferencia um grupo humano de outro. Para Schein (1992), cultura é um fenômeno que envolve todos, a todo o momento, sendo constantemente representada e criada por meio das interações entre os indivíduos. Trata-se de um processo dinâmico, evolutivo e interativo. Ao se referir a este aspecto Bhagwati (2004) ressalta que as culturas se enriquecem com as importações culturais formando um grande mosaico.

Keegan e Green (1999, p.92) complementam afirmando que: "A cultura determina o modo de vida do homem no contexto de instituições sociais, instituições educacionais, religiosas, governamentais e de negócios. Compreende valores, idéias, atitudes e símbolos conscientes e inconscientes que moldam o comportamento humano e que são transmitidos de geração em geração".

Já no entendimento de Cobra (1995, p.74), a cultura é entendida como um "conjunto de crenças, valores, linguagem, religião, arte, moral, leis, educação, costumes, hábitos e capacidade de aprendizado de membros de uma sociedade". Observa-se tanto nos conceitos de Cobra (1995) quanto no de Keegan e Green (1999) que a cultura apresenta uma variedade de elementos que mesclados formam uma identidade cultural.

A cultura apresenta várias dimensões e a sua compreensão contribui para que gestores, que atuam em mercados internacionais, possam melhor entender a extensão em que a cultura pode afetar os seus negócios. No entendimento de Johanson e Vahlne (1977), o desconhecimento das diferenças culturais entre os países tem se tornado um obstáculo para o desenvolvimento de operações internacionais. As diferenças culturais entre os países constituem-se no principal elemento diferenciador entre operações internacionais e domésticas.

Calcula-se que mais de $50 \%$ dos negócios internacionais não são concluídos pelo desconhecimento das diferenças culturais. Essas, por sua vez, são partes fundamentais da gestão do mercado internacional, ou seja, o conhecimento do idioma, entender a cultura local, conhecer as técnicas comerciais, e buscar a sintonia com o parceiro o qual se pretende negociar (MINERVINI, 2001).

O conceito de distância psíquica é elemento importante quando se analisa a influência da cultura nos negócios internacionais. Johanson e Vahlne (1977) destacam que distância psíquica é a soma de fatores que atrapalham o fluxo de informação de um mercado para o outro, como as diferenças na linguagem, na educação, nas práticas administrativas, na cultura e no desenvolvimento industrial. Estes fatores fazem com que a complexidade de se atuar nos mercados internacionais seja mais elevada do que no âmbito doméstico, devido ao maior número de elementos que interferem no processo de comunicação entre partes localizadas em diferentes países. 
Ao analisar organizações que estão iniciando seu processo de internacionalização, Faulkner (2003) enfatiza que há uma tendência de realizar negócios com empresas localizadas em países culturalmente semelhantes aos seus. Isto pode ser explicado pelo fato de se acreditar que o nível de incerteza aumenta com o aumento da distância psíquica. Para Hilal e Hemais (2003), as firmas têm melhor conhecimento de seus ambientes mais imediatos, assim elas procuram alternativas em que se sintam o menos estrangeiras possível.

Meyer Jr., Meyer e Murphy (2006) corroboram afirmando que o medo de um choque cultural é tão grande que as empresas buscam iniciar seus empreendimentos internacionais em países culturalmente semelhantes aos seus. No entendimento dos autores é comum observar empresas norte-americanas iniciarem sua internacionalização nos mercados do Canadá ou da Grã-Bretanha, em razão da semelhança cultural. Da mesma forma, empresas argentinas se inserem internacionalmente a partir dos mercados uruguaio, paraguaio ou chileno.

A importância das diferenças culturais nos negócios internacionais é grande, mas essas variáveis não são facilmente identificáveis em uma análise superficial. Muitas vezes as pessoas não se dão conta do que consiste a sua própria cultura. Segalla (2001) destaca que poucas pessoas realmente conhecem os seus próprios valores culturais até terem os mesmos ameaçados por outras culturas. Para o mesmo autor, esta é a razão pela qual muitos programas de integração internacional realizados por empresas, com operações em diferentes países, muitas vezes fracassam.

O sucesso da operação internacional de uma organização irá depender da capacidade de adaptar suas operações, seus produtos e serviços à realidade do país hospedeiro. Kogut (2002) argumenta que a empresa que atua em vários países precisa desenvolver flexibilidade para se adaptar às exigências dos mercados nacionais. Rowley e Benson (2002), em estudo sobre administração de recursos humanos em quatro diferentes países asiáticos, concluíram que é difícil universalizar as práticas de gerenciamento de pessoas em diferentes países devido a variáveis culturais e torna-se necessário tratar cada país de forma individualizada.

Ao pesquisarem o impacto das diferenças culturais em empresas multinacionais holandesas, Drogendijk e Slangen (2006) reforçam o entendimento de que a distância cultural é um fator dificultador na instalação de empresas multinacionais em países estrangeiros independentemente da experiência internacional ou tamanho da empresa.

A observância dos vários elementos culturais é uma condição imperiosa para o êxito nos negócios internacionais. As facilidades geradas pela conectividade e a rapidez das informações podem auxiliar o gestor a desenvolver uma maior compreensão sobre as diferentes culturas e, conseqüentemente, encontrar maneiras de viabilizar o seu empreendimento.

\subsection{Internacionalização da Educação Superior}

Uma das conseqüências do movimento de globalização é justamente a internacionalização de organizações. Cada vez mais empresas de todo o mundo estão internacionalizando as suas atividades, tirando vantagem de oportunidades em mercados externos. 
Na concepção de Reitter e Chassang (2001, p.174), "uma empresa quando se torna internacional, não muda somente de natureza, mas também sua complexidade. A organização adquire mais diversidade, e por conseqüência busca ter mais unidade”. Este conceito ilustra a necessidade das organizações mudarem o seu comportamento nos mercados internacionais para se tornarem mais competitivas. Isto, no entanto, aumenta a complexidade organizacional na medida em que a torna mais heterogênea.

As instituições de educação superior começam a tirar proveito desse novo contexto trazido pela globalização. Já se observa uma crescente expansão das atividades dessas instituições em outros países e continentes, em busca de novos mercados e novas oportunidades de negócios. O resultado desta expansão é o aumento da concorrência e da oferta de cursos em todo o mundo (MEYER JR.; MEYER, 2006).

Conseqüentemente, várias instituições de educação superior estão atuando em países diferentes daqueles onde tinham originalmente as suas atividades. Muitas das instituições de ensino superior que se internacionalizaram, começaram fazendo pequenos investimentos em países vizinhos.

Ao abordarem a expansão internacional da educação superior, Meyer Jr. e Mugnol (2004) ressaltam a presença de empresas educacionais no cenário global citando os exemplos do grupo Apollo, ligado a University of Phoenix, do grupo Sylvan Learning Systems, da Devry Incorporated e da Career Education Corporation, que atuam em várias partes do mundo explorando a educação em geral e, a educação superior, em particular.

Outra organização que possui uma presença intensa nos vários países do Reino Unido é a Open University. Reconhecida por seu pioneirismo e experiência na área da educação à distância e da oferta de cursos presenciais a Open University tem expandindo suas atividades no mercado competitivo da educação superior do Reino Unido.

Em face desta expansão internacional das instituições de educação superior, começam a haver disputas internacionais por fatias de mercado. A University of Phoenix, por exemplo, ingressou no mercado da Grã-Bretanha, mas não conseguiu desenvolver estratégias capazes de suplantar a Open University em seu próprio território. O que acabou levando a universidade americana a se retirar da Grã-Bretanha (DOUGLASS, 2005). Já na América Latina, observa-se a significativa expansão da Laureate Education Inc. com presença em países como México, Honduras, Panamá, Costa Rica, Equador, Peru e Brasil, criando uma verdadeira rede de universidades.

A internacionalização de instituições de educação superior tem levado as instituições locais a reexaminarem o seu gerenciamento, em especial no que se refere ao posicionamento estratégico, marketing e oferta de programas acadêmicos diferenciados. Algumas universidades têm se utilizado de novas tecnologias para ofertar os seus produtos e serviços educacionais alcançando novos mercados.

\subsection{Estratégias}

Atraídas por novos e promissores mercados e pelo potencial de rentabilidade do setor educacional, grupos econômicos e empresas educacionais passaram a expandir suas atividades desenvolvendo estratégias para viabilizar os novos empreendimentos em países e culturas 
distintos. As estratégias se transformam em ingredientes importantes para orientar os esforços internacionais das empresas e inúmeras são as formas utilizadas. Parker (1999) destaca que o mundo globalizado tem exigido novas abordagens para a formulação da estratégia em comparação com os modelos tradicionais.

A estratégia, para Ansoff (1988), é um conjunto de regras para a tomada de decisão que orientam o comportamento organizacional. Estratégia é também vista como um mecanismo que guia ou orienta o alinhamento com o ambiente externo assim como promove a integração interna dos diversos setores e operações (SNOW; HAMBRICK, 1980). Ohmae (1982), por sua vez, faz uma analogia entre o mundo dos negócios e o campo de batalha, enfatizando que o objetivo da estratégia é criar condições favoráveis, julgando o melhor momento para atacar ou se retirar.

Mintzberg (1994) destaca que a estratégia pode apresentar vários significados. Estratégia pode ser vista como um plano, uma direção, um guia ou curso de ação para o futuro, ou como um padrão, uma consistência de comportamento de uma organização ao longo do tempo. Estratégia pode significar também uma posição que caracteriza determinada organização, ou a definição de seus produtos em relação ao mercado. Finalmente, estratégia pode significar uma perspectiva, ou seja, a maneira de a organização fazer as coisas.

Para se atingir seus objetivos, as organizações se utilizam de estratégias competitivas ou colaborativas. As estratégias competitivas são aquelas nas quais se imaginam firmas independentes tentando derrotar outras empresas. Já as colaborativas são utilizadas quando empresas tentam desenvolver vantagens competitivas por meio de colaboração com outras empresas (BARNEY, 1997).

Analisando as estratégias competitivas, pode-se definir o seu objetivo de acordo com a visão de Tallman (2003, p.373) de "colocar os produtos e serviços da empresa em um nicho de mercado que pode ser isolado do excesso de competição e proporcionar lucros acima da média”. Ao examinarem as estratégias colaborativas, Kluyver e Pierce II (2007) destacam que as mesmas tornaram-se muito populares nos últimos anos, já que capturam os benefícios do desenvolvimento interno e da aquisição ao mesmo tempo em que evitam as desvantagens de ambos. Esses mesmos autores ressaltam como principais formas de estratégias colaborativas, as joint ventures e as alianças estratégicas.

Observa-se que as estratégias competitivas e colaborativas são distintas no sentido que percebem seus concorrentes de forma diferenciada. Na primeira, o concorrente é encarado com desconfiança e como alguém que precisa ser vencido. Já na segunda, o concorrente é visto como um aliado em potencial de quem se pode aprender ou desenvolver algo em conjunto.

As empresas utilizam-se das abordagens competitiva e colaborativa para atingir os seus objetivos. Estas podem ser utilizadas separadamente ou de forma integrada para fortalecer a posição da empresa em um determinado mercado.

O sucesso das estratégias de organizações que atuam em diferentes países está associado a sua capacidade de aprendizagem, como reforça Parker (1999). Uma organização que é eficaz em sua aprendizagem será tanto capaz de criar, adquirir e transferir conhecimento, assim como modificar o seu comportamento para refletir este novo conhecimento (CHILD, 2003). 
Organizações que atuam em mercados internacionais estão constantemente sendo desafiadas pelo dilema de utilizar-se de estratégias locais ou globais. Sobre este assunto, Buckley e Ghauri (2004) argumentam que é necessário ter flexibilidade ao combinar estratégias globais com locais, de forma a encontrar o equilíbrio certo para cada mercado. $\mathrm{Na}$ visão dos mesmos autores, as diferenças culturais apresentam uma grande importância na determinação deste equilíbrio de estratégias.

\section{METODOLOGIA}

Este trabalho caracteriza-se como um estudo de caso descritivo. Trata-se de uma análise detalhada da experiência recente da Universidad de la Integración de las Américas UNIDA - estabelecida, em Assunção, no Paraguai.

Para Yin (1993), o estudo de caso descritivo apresenta uma completa descrição de um fenômeno inserido em seu contexto estabelecendo relações de causa e efeito. Na percepção de Remenyi et al (1998), os estudos de caso permitem explorar as características de eventos da vida real das organizações como processos gerenciais, maturação de setores ou disputas de poder. Trata-se, segundo os mesmos autores, de um excelente instrumento de desenvolvimento gerencial ao combinar ação e conhecimento.

Os dados e informações relativas às ações gerenciais da UNIDA foram coletados por meio de questionário semi-estruturado, com questões abertas, aplicado à totalidade dos gestores estratégicos da instituição foco do estudo, ou seja, o diretor geral, o diretor financeiro e o diretor acadêmico. Além das entrevistas com os dirigentes da instituição foi também entrevistado um ex-dirigente da Universidade, que se constituiu em respondente-chave por seu conhecimento e experiência na gestão da instituição e que compartilhou suas anotações pessoais enquanto diretor da Universidade. Nas entrevistas buscou-se obter a percepção dos respondentes acerca das estratégias utilizadas e os obstáculos de natureza cultural enfrentados. O estudo abrangeu os anos de 2003-2006 que corresponde ao período referente à instalação e primeiros anos de funcionamento da instituição.

A UNIDA é uma instituição privada de educação superior paraguaia que iniciou suas atividades em 2003, resultante de investimento internacional do grupo brasileiro Estácio de Sá. A Universidade localiza-se na cidade de Assunção onde oferece 12 cursos de graduação além de diversos cursos de especialização e de extensão à comunidade. Atualmente, a instituição possui um corpo discente de 1.800 alunos estando em franca expansão de suas atividades. A UNIDA destaca-se pelos seguintes fatores: qualidade da educação ofertada, localização das suas instalações, diversidade dos seus cursos, agilidade com que responde as demandas do mercado e ações comunitárias que a tornam uma das destacadas instituições do país.

\section{DISCUSSÃO E ANÁLISE DOS DADOS}

A chegada da UNIDA no mercado da educação superior paraguaio representou um marco no setor com a introdução de práticas gerenciais, com destaque para ações de marketing, que a diferenciaram das demais instituições. A UNIDA, desde o início de suas atividades, no ano de 2003, procurou se distinguir das demais universidades do mercado local 
adotando práticas inovadoras, muitas delas transplantadas da rede de instituições de ensino do grupo Estácio de Sá no Brasil.

Os dirigentes entrevistados afirmaram que a entrada no mercado paraguaio levou a UNIDA a desenvolver sete estratégias principais para firmar seu posicionamento. Essas estratégias estão relacionadas à localização, currículos acadêmicos, propaganda, ações de responsabilidade social, infra-estrutura, aliança estratégica, escolha da liderança e políticas de intercâmbio com o Brasil.

\begin{tabular}{|l|l|l|l|}
\hline & Estratégia & Tipo (Barney, 1997) & Elemento Chave \\
\hline 1. & Localização do Campus & Colaborativa & $\begin{array}{l}\text { Proximidade ao centro urbano de } \\
\text { Assunção }\end{array}$ \\
\hline 2. & Programas acadêmicos & Competitiva & Currículo inovador \\
\hline 3. & Propaganda diferenciada & Competitiva & Ações inovadoras diferenciadas \\
\hline 4. & Responsabilidade social & Competitiva & $\begin{array}{l}\text { Programas relevantes à } \\
\text { comunidade }\end{array}$ \\
\hline 5. & Infraestrutura & Colaborativa & Qualidade das instalações \\
\hline 6. & Liderança & Competitiva & $\begin{array}{l}\text { Escolha de líder político local } \\
\text { como Reitor }\end{array}$ \\
\hline 7. & Intercâmbio & Competitiva & Uso da Rede Estácio de Sá \\
\hline
\end{tabular}

Quadro 1 - Estratégias utilizadas pela UNIDA, segundo seus dirigentes.

Fonte: dados primários

A localização da Universidade, em Assunção, próxima ao centro da cidade, constituise em fator de grande importância para o sucesso do empreendimento. Essa estratégia representa um diferencial competitivo da Universidade em relação aos seus grandes concorrentes como a Universidad Nacional de Asunción (UNA) e a Universidad Católica, cujos campi estão localizados em áreas distantes do centro de Assunção. Ainda para reforçar o aspecto da localização, a instituição colocou à disposição dos alunos, ônibus gratuitos para transporte entre a Universidade e cidades da área metropolitana da capital paraguaia.

Uma segunda estratégia identificada pelos entrevistados foi a reformulação dos programas acadêmicos ofertados pela instituição. A UNIDA criou uma estrutura curricular inovadora baseada no sistema curricular que o grupo Estácio de Sá utiliza no Brasil. Os currículos dos cursos oferecidos procuraram refletir as reais necessidades do mercado, eliminando assuntos desatualizados e pouco utilizados. Esta estratégia facilitou também o intercâmbio de alunos entre as instituições integrantes do grupo fortalecendo sua internacionalização. Percebe-se nesta estratégia, a preocupação de se adequar os programas acadêmicos a realidade sócio-econômico-cultural local, revelando uma necessária flexibilidade, como enfatizam Rowley e Benson (2002), assim como Kogut (2002).

A propaganda constituiu-se na terceira estratégia empregada pela UNIDA na percepção dos seus dirigentes. Nessa área, a Universidade adotou uma postura agressiva e inovadora que resultou em práticas miméticas dos seus concorrentes. A instituição foi a primeira universidade do país a fazer uso do busdoor, que trouxe claros resultados na demanda por seus cursos. A UNIDA, por ser um novo ator no mercado paraguaio da educação superior, optou por adotar uma estratégia com forte conteúdo publicitário de forma a difundir a sua marca. Outros instrumentos de mídia e promoção foram utilizados com sucesso, como 
comunicação via rádio AM e FM, outdoors em ônibus, panfletagem e, ainda, anúncios em jornais de grande circulação, como ABC Color e Última Hora.

A quarta estratégia materializou-se em ações de responsabilidade social desenvolvidas pela instituição. A estratégia foi oferecer à comunidade cursos gratuitos de férias em diversas áreas técnicas, relevantes à atividade econômica local, possibilitando a ampliação de conhecimentos e incremento na renda familiar. A participação nos cursos foi condicionada a doação de alimentos não perecíveis. Uma segunda ação foi a doação dos alimentos arrecadados pela instituição à entidades filantrópicas. Os cursos geraram uma demanda de aproximadamente mil participantes. Essas ações contribuíram para tornar a UNIDA uma instituição mais conhecida na comunidade, fortalecendo seu compromisso social.

A diferenciação por meio de infra-estrutura moderna e adequada ao processo ensino aprendizagem constituiu-se na quinta estratégia organizacional. A infra-estrutura é uma das vantagens competitivas da UNIDA por ser reconhecida pela comunidade como a Universidade com as melhores instalações do Paraguai. A instituição possui invejável infraestrutura, por estar instalada em um edifício vistoso, moderno, bem estruturado, com salas de aula climatizadas, biblioteca, laboratórios de informática e laboratórios específicos para determinadas áreas da saúde, constituindo-se em fator de atratividade de alunos. O Complexo Barrail, edifício onde se localiza a UNIDA, diferencia-se pela sua beleza e limpeza, fatores esses que não são presentes na maioria das demais instituições de educação superior do país. Esta estratégia constituiu-se em vantagem comparativa para UNIDA, contribuindo para a expansão da demanda dos seus diversos cursos.

A escolha do líder constitui-se na sexta estratégia adotada pela UNIDA. Foi escolhido para ocupar o cargo de reitor da Universidade, um ex-senador e ex-vice-presidente do Senado paraguaio. Essa escolha reflete uma estratégia simbólica de inserção da organização na sociedade do Paraguai. O prestígio pessoal do reitor transfere-se à instituição, não apenas internamente, mas, principalmente, no âmbito político, presente nas relações interinstitucionais, proporcionando maior legitimidade à Universidade. Esta estratégia serviu para reduzir a distância psíquica entre a instituição de capital brasileiro e a comunidade paraguaia. Isto reforça o pensamento de Johanson e Vahlne (1977) quanto à importância da leitura de um contexto para redução de barreiras objetivando um melhor desempenho organizacional.

A sétima estratégia foi a utilização da rede do grupo Estácio de Sá, no Brasil, para intercâmbio de alunos. Os convênios e parcerias com várias instituições brasileiras constituíram-se em importante diferencial. O Brasil desfruta de uma imagem positiva no Paraguai e essa tem sido uma das fortes razões pelas quais jovens paraguaios anseiem estudar em universidades brasileiras. Os convênios com a rede de instituições de educação superior que integram a rede Estácio de Sá constituíram-se em fator de atração de alunos e diferenciação no mercado, caracterizando uma estratégia colaborativa típica, como destacado por Kluyver e Pierce II (2007).

Observa-se, assim que as estratégias utilizadas pela UNIDA se assemelham àquelas apontadas por Barney (1997). Em processos de internacionalização é importante haver uma combinação dos dois tipos de estratégia referidos pelo autor. Este estudo revela que foram utilizadas tanto estratégias competitivas quando colaborativas visando o desenvolvimento de 
vantagem competitiva no mercado paraguaio da educação superior. Percebe-se, claramente, a predominância das estratégias competitivas no estudo de caso analisado, conforme demonstra o quadro 1 .

\subsection{Impacto Cultural}

A implantação da UNIDA, no mercado paraguaio, foi acompanhada de um impacto cultural. Vários fatores contribuíram para o desenvolvimento deste quadro. Destaca-se que, inicialmente, a instalação da Universidade gerou uma expectativa positiva na comunidade local por se tratar de um grupo brasileiro com um projeto educacional voltado ao ensino superior de qualidade.

Reforça este cenário a visão positiva que os paraguaios têm do Brasil. É conhecida a influência brasileira no país vizinho, não só pela dimensão geopolítica ocupada pelo país na América Latina, mas, principalmente, pelo tamanho e pujança da sua economia, tamanho do seu mercado, oportunidades de negócios, infra-estrutura de serviços, qualidade da educação e pelo nível de desenvolvimento científico e tecnológico atingido pelo Brasil. Reforçam esta posição os inúmeros negócios, parcerias, acordos e convênios existentes envolvendo organizações de ambos os países. O Mercado Comum do Cone Sul - MERCOSUL também contribui decisivamente para a maior aproximação entre os dois países.

A experiência da UNIDA revela que o "estilo Estácio de Sá" de gerenciar foi introduzido com sucesso. Ao se referir aos benefícios gerados por empresas atuando no exterior, Bhagwati (2004) enfatiza novas abordagens gerenciais que introduzidas por essas empresas e que passam a ser incorporadas localmente beneficiando diferentes grupos. Contudo, diversos problemas surgiram durante os primeiros anos de funcionamento da UNIDA no Paraguai. Inicialmente, as maiores dificuldades enfrentadas pela instituição estavam relacionadas ao fato de seus gestores introduzirem, sem maior adaptação, abordagens de gestão acadêmica utilizadas, com sucesso, pela rede do grupo Estácio de Sá no Brasil. Muitas dessas práticas gerenciais não estavam em sintonia com a cultura paraguaia. Como exemplo, pode-se destacar: a denominação dos cursos, a utilização de tecnologia em processos acadêmicos, o modelo de realização de provas em dias consecutivos, a exigência de cumprimento de horário do corpo docente e a oferta de algumas disciplinas incompatíveis com a realidade do país.

A UNIDA implantou uma política de contratação de professores e funcionários centrada na seleção de profissionais brasileiros, residentes no Paraguai. Uma possível razão para adoção desta política, segundo um dos entrevistados, foi a busca de uma maior coesão interna na instituição, especialmente em sua fase inicial, contratando profissionais de um mesmo background cultural. Contudo, esta política provocou um novo problema cultural ao criar, na UNIDA, um clima de divisão entre brasileiros e paraguaios.

Outro elemento cultural importante é o idioma e esse foi um dos principais fatores que contribuiu para a dificuldade de coesão, enfraquecendo a cultura organizacional em formação. Muitos professores e gestores brasileiros apresentavam limitações em falar e entender o idioma espanhol, gerando na comunidade interna da UNIDA dificuldades de comunicação, mau entendimento, frustração e problemas de gestão. Soma-se a esse problema o fato dos 
primeiros diretores geral e acadêmico da instituição serem brasileiros e não terem o domínio da língua espanhola.

O etnocentrismo de alguns funcionários e professores brasileiros foi outro fator apontado pelos entrevistados como agravante na divisão entre brasileiros e paraguaios que integravam a comunidade da UNIDA. Este comportamento foi reforçado por atitudes dos gestores que sempre enfatizavam que "as coisas deveriam ser feitas tais como são realizadas no Brasil". A comunidade da UNIDA tinha a percepção que por detrás de muitas das decisões e ações, nas áreas acadêmica e administrativa, estava embutida a crença de uma superioridade brasileira em relação à realidade paraguaia.

Aspectos comportamentais como a falta de pontualidade do corpo docente foi outro fator cultural encontrado, já que os professores universitários paraguaios não têm a cultura de cumprimento de horários de aula. Habituados ao sistema público de ensino superior do país, até então predominante, os docentes não consideravam o horário com a devida importância, fazendo com que, muitas vezes, as aulas terminassem mais cedo ou começassem atrasadas. A rigidez imposta pela UNIDA na exigência do cumprimento do horário das aulas provocou conflitos entre professores e direção.

Acostumados, por razões culturais, a não reprovação, os alunos da UNIDA resistiram às reprovações nas disciplinas. Até então, os alunos no Paraguai estavam acostumados a se submeterem a provas sucessivas até atingirem o nível de aprovação. Preocupados com o nível de qualidade de ensino, a Universidade reexaminou sua política de aplicação de provas finais, aumentando para duas as chances de recuperação. Essa foi uma decisão que a instituição entendeu como não comprometedora da qualidade do ensino. Ainda assim, existiram atritos e reclamações por parte dos alunos. A preocupação em flexibilizar as estratégias da instituição, para se adaptar a cultura local, corrobora o argumento apresentado por Buckley e Ghauri (2004).

A inexistência de uma tradição de educação superior no Paraguai e o fato deste setor ser pouco regulamentado contribuiu, favoravelmente, para a instalação e o desenvolvimento da UNIDA, da mesma forma que tem facilitado a entrada de outras instituições estrangeiras. Atualmente, este mercado caracteriza-se por três importantes aspectos: o primeiro refere-se ao crescente número de instituições de educação superior que se instalam no país, muitas delas ligadas a grupos estrangeiros. O segundo destaca a diversidade dos níveis de qualidade de educação oferecida pelas instituições. Finalmente, o terceiro, reforça a acirrada competição existente entre as instituições que buscam conquistar novos nichos de mercado, utilizando, para isto, uma gama ampla de estratégias.

As diferenças culturais, econômicas e sociais entre países e a dificuldade de se "commoditizar" a educação tem dificultado, sobremaneira, a internacionalização de grupos educacionais no mundo. Uma razão plausível para explicar a inabilidade em lidar inicialmente com os aspectos culturais, demonstrada pela gestão da Universidade, pode ser atribuída ao fato dos seus gestores não possuírem experiência na gestão de negócios internacionais. Drogendijk e Slangen (2006), por sua vez, já destacavam em seu estudo, que a experiência internacional dos gestores nem sempre diminui a distância cultural em empreendimentos no exterior. 
O caso da UNIDA é representativo de um esforço empreendedor bem sucedido de um grupo brasileiro que, de forma inédita, decidiu expandir suas atividades no exterior na área da educação superior. Esta experiência permitiu ao grupo Estácio de Sá criar, adquirir e transferir conhecimento em sua rede de instituições. Isto vem ao encontro do que destaca Child (2003) ao se referir à importância aprendizado organizacional para o sucesso de qualquer esforço inovador. Os negócios internacionais no Paraguai serviram de estímulo ao grupo Estácio de Sá para expandir suas atividades no exterior ao adquirir o controle do Instituto Universitario Autônomo del Sur, no Uruguai.

Um dos maiores desafios para a gestão da UNIDA foi adequar as políticas e estratégias da organização à cultura local. Uma vez que dois terços dos seus dirigentes eram brasileiros e não tinham maior experiência em negócios internacionais, o processo de adaptação foi incremental e iterativo, mas permitiu, graças à habilidade dos gestores, que a instituição atingisse seus objetivos, nos primeiros anos de atuação, no exterior.

\section{CONCLUSÕES}

A decisão de instalar qualquer instituição de educação superior no exterior implica em desafios que, por sua vez, requerem ações estratégias adequadas para a viabilização do empreendimento. Trata-se de uma iniciativa complexa por abarcar diversos fatores como o econômico, o político-legal e o cultural com implicações na gestão estratégica e operacional da organização.

A análise da experiência da Universidade de la Integración de las Américas, no Paraguai, revelou resultados positivos. Destaca-se a visão estratégica do grupo Estácio de Sá que encontrou no país vizinho um mercado em crescimento onde a educação superior é recente e pouco regulamentada.

A utilização de estratégias adotadas pela UNIDA contribuiu, decisivamente, para a viabilização do empreendimento no Paraguai. Um dos maiores desafios foi a adequação dessas estratégias à cultura local. A flexibilidade gerencial e capacidade adaptativa da Universidade constituíram-se em fatores decisivos nesta adequação.

Foi observada, no início do processo de internacionalização, certa negligência da gestão da UNIDA quanto a aspectos culturais locais. Isto ficou evidente na introdução de abordagens gerenciais importadas do modelo "Estácio de Sá" praticadas no Brasil. Esse fato gerou conflitos e dificuldades gerenciais, os quais foram posteriormente sanados. Essa negligência identificada pode ser atribuída à inexperiência internacional do grupo gestor responsável pelo empreendimento.

A resistência é uma conseqüência natural em qualquer processo de mudança ou inovação. No entanto, é necessário, dentro de qualquer processo de internacionalização, observar se as mudanças e novidades que estão sendo introduzidas estão em conformidade com as regras culturais da localidade onde se atua.

O caso da UNIDA revela um exemplo bem sucedido de internacionalização de um grupo de educação superior brasileiro. Essa experiência revela habilidade gerencial no desenvolvimento de estratégias que permitiram a viabilização do empreendimento. Por outro 
lado, dificuldades iniciais resultantes de conflitos culturais serviram de aprendizado aos seus gestores na administração de uma instituição de educação superior no âmbito internacional.

\section{REFERÊNCIAS}

ANSOFF, H. I. The new corporate strategy. New York: John Wiley \& Sons, 1988.

BUCKLEY, P. J.; GHAURI, P. N. Globalisation, economic geography and the strategy of multinational enterprises. Journal of International Business Studies. East Lansing, n. 35, p. 81-98, 2004.

BARNEY, J. B. Gaining and sustaining competitive advantage. New York: AddisonWesley, 1997.

BASSI, E. Globalização de negócios. São Paulo: Cultura, 1997.

BHAGWATI, J. In defense of globalization. New York: Oxford University Press, 2004.

CHILD, J. Organizational learning. In: FAULKNER, D. O.; CAMPBELL, A. The Oxford handbook of strategy, volume I: a strategy overview and competitive strategy. New York: Oxford University Press, 2003. v. 1. p. 437-465.

COBRA, M. Ensaio de marketing global. São Paulo: Marcos Cobra Editora, 1995.

DOUGLASS, J. A. How all globalization is local: countervailing forces and their influence on higher education markets. Higher Education Policy. Hampshire, n.18, 2005, p. 445-473.

DROGENDIJK, R.; SLANGEN, A. Hofstede, Schwartz, or managerial perceptions? The effects of different cultural distances measures on establishment mode choices by multinational enterprises. International Business Review. Manchester, n.15, 2006, p. 361380 .

FAULKNER, D. International strategy. In: FAULKNER, D. O.; CAMPBELL, A. The Oxford handbook of strategy, volume II: corporate strategy. New York: Oxford University Press, 2003, p. 159-182.

GRAY, J. Falso amanhecer: os equívocos do capitalismo global. Rio de Janeiro: Record, 1999.

HILAL, A.; HEMAIS, C. A. O processo de internacionalização na ótica da escola nórdica: evidências empíricas em empresas brasileiras. Revista de Administração Contemporânea. Curitiba, v.7, n.1, jan./mar. 2003, p. 109-124.

HOFSTEDE, G. Culture's consequences: international differences in work related values. Beverly Hills: Sage Publications, 1984. 
JOHANSON, J.; VAHLNE, J. The internationalization process of the firm: a model of knowledge development and increasing foreign market commitments. Journal of International Business Studies. Hampshire, n.8, spring, 1977, p. 23-32.

KEEGAN, W. J.; GREEN, M. C. Princípios do marketing global. São Paulo: Saraiva, 1999.

KLUYVER, C. A.; PIERCE II, J. A. Estratégia: uma visão executiva. São Paulo: Pearson Prentice Hall, 2007.

KOGUT, B. International management and strategy. In: PETTIGREW, A.; THOMAS, H.; WHITTINGTON, R. Handbook of strategy and management. London: Sage Publications, 2002, p. 261-278.

MEYER JR., V; MEYER, B. La globalización da la educación superior. Visiones de la Educación. Concepción, n.10, 2006, p.77-87.

MEYER JR., V.; MEYER, B.; MURPHY, J. P. The influence of culture in international business. In: ENCONTRO DA ASSOCIAÇÃO NACIONAL DE PÓS-GRADUAÇÃO E PESQUISA EM ADMINISTRAÇÃO, 30, 2006, Salvador. Anais... Salvador, 2006.

MEYER JR., V.; MUGNOL, G. Competição e estratégias no contexto das instituições de ensino superior privadas. Revista Diálogo Educacional. Curitiba, jan./abr. 2004, p. 153-165.

MINERVINI, N. O exportador: ferramentas para atuar com sucesso nos mercados internacionais. 3. ed. São Paulo: Pearson Education do Brasil, 2001.

MINTZBERG, H. The rise and fall of strategic planning. New York: Free Press, 1994.

NAÍM, M. Ilícito: ataque da pirataria, da lavagem de dinheiro e do tráfico à economia global. Rio de Janeiro: Jorge Zahar, 2006.

OHMAE, K. The mind of the strategist. New York: McGraw-Hill, 1982.

OHMAE, K. O novo palco da economia global: desafios e oportunidades em um mundo sem fronteiras. Porto Alegre: Bookman, 2006.

PARKER, B. Evolução e revolução: da internacionalização a globalização. In: CLEGG, S. R.; HARDY, C.; NORD, W.R. Handbook de Estudos Organizacionais, volume I: modelos de análise e novas questões em estudos organizacionais. São Paulo: Atlas, 1999, p. 400-433.

REITTER, R.; CHASSANG, G. Manobrando o caos e a tirania. In: Dominando mercados globais. São Paulo: Makron Books, 2001. p. 174-178.

REMENYI, D. et al. Doing research in business and management. London: Sage Publications, 1998.

ROWLEY, C.; BENSON, J. Convergence and divergence in Asian human resource management. California Management Review. Berkeley, v.44, n.2, winter, 2002, p. 90-109.

SCHEIN, E. H. Organizational culture and leadership. Jossey-Bass: San Francisco, 1992. 
SEGALLA, M. Culturas nacionais, negócios internacionais. In: Dominando mercados globais. São Paulo: Makron Books, 2001. p. 164-169.

SNOW, C. C.; HAMBRICK, D. C. Measuring Organizational Strategies: some theoretical and methodological problems. Academy of Management Review, v. 5, n. 4, 1980, p. 527538.

TALLMAN, S. Dynamic Capabilities. In: FAULKNER, D. O.; CAMPBELL, A. The Oxford handbook of strategy, volume I: a strategy overview and competitive strategy. New York: Oxford University Press, 2003. v. 1. p. 372-403.

YIN, R. K. Applications of case study research. Newbury Park: Sage Publications, 1993. 